\title{
Somatosensory information processing in the aging population
}

\section{Zheng Zhang, Eric M. Francisco, Jameson K. Holden, Robert G. Dennis and Mark Tommerdahl*}

Department of Biomedical Engineering, University of North Carolina, Chapel Hill, NC, USA

\section{Edited by:}

Hari S. Sharma, Uppsala University, Sweden

\section{Reviewed by:}

Junming Wang, University of

Mississippi Medical Center, USA

Jiawei Zhou, Shanghai Institutes for

Biological Sciences of Chinese

Academy of Sciences, China

\section{${ }^{*}$ Correspondence:}

Mark Tommerdahl, Department of Biomedical Engineering, University of North Carolina, CB \#7575, Chapel Hill, NC 27599, USA.

e-mail: tommerda@med.unc.edu
While it is well known that skin physiology - and consequently sensitivity to peripheral stimuli - degrades with age, what is less appreciated is that centrally mediated mechanisms allow for maintenance of the same degree of functionality in processing these peripheral inputs and interacting with the external environment. In order to demonstrate this concept, we obtained observations of processing speed, sensitivity (thresholds), discriminative capacity, and adaptation metrics on subjects ranging in age from 18 to 70 . The results indicate that although reaction speed and sensory thresholds change with age, discriminative capacity, and adaptation metrics do not. The significance of these findings is that similar metrics of adaptation have been demonstrated to change significantly when the central nervous system (CNS) is compromised. Such compromise has been demonstrated in subject populations with autism, chronic pain, acute NMDA receptor block, concussion, and with tactile-thermal interactions. If the metric of adaptation parallels cortical plasticity, the results of the current study suggest that the CNS in the aging population is still capable of plastic changes, and this cortical plasticity could be the mechanism that compensates for the degradations that are known to naturally occur with age. Thus, these quantitative measures - since they can be obtained efficiently and objectively, and appear to deviate from normative values significantly with systemic cortical alterations - could be useful indicators of cerebral cortical health.

Keywords: aging, sensory, plasticity, adaptation, tactile, somatosensory

\section{INTRODUCTION}

There have been a number of significant findings related to both the anatomical and physiological degradation that occurs with normal aging. For example, structural and functional neuroimaging studies have consistently shown evidence of age-related reduction of cerebral cortical volume (Resnick et al., 2003; Raz et al., 2005; Driscoll et al., 2009; Fjell et al., 2009) and changes of white matter integrity in healthy older adults (Gunning-Dixon and Raz, 2000; Bartzokis et al., 2003; Gunning-Dixon et al., 2009). However, a number of researchers have noted that cognitive performance is relatively stable with normal aging (Morse, 1993; Wilson et al., 2002; Van Petten et al., 2004), although some metrics of sensory performance (e.g., thresholds) degrade (Verrillo, 1982; Gescheider et al., 1994; Verrillo et al., 2002; Lin et al., 2005). Dinse made the observation that restoration of function in the aging population is attainable due to the emergence of new processing strategies, and he attributed this to brain plasticity being operational in the aging population (Dinse, 2006). In a recent review, Greenwood put forth a hypothesis that with aging, although there is significant evidence of both anatomical and physiological decline, there is no, or even negative, correlation with cognitive performance. Greenwood largely attributes the undefined compensatory mechanism that allows for maintenance of cortical information processing capacity to cortical plasticity (Greenwood, 2007; Greenwood and Parasuraman, 2010).

Recently, we have developed unique sensory based measures that quantify particular aspects of a subject's central information processing capacity (Tannan et al., 2005a,b, 2006, 2007a,b, 2008; Tommerdahl et al., 2007a,b, 2008; Folger et al., 2008; Francisco et al., 2008; Zhang et al., 2008, 2009, 2011). One particular focus of these studies has been on obtaining measures of centrally mediated adaptation - a process that is a fundamental component of cortical plasticity and operates on multiple time scales (for review, see Kohn, 2007). If cortical plasticity is the mechanism by which cortical information processing capacity is maintained, and if adaptation does, in fact, parallel cortical plasticity, then we would predict that metrics of adaptation would remain constant with normal aging. In terms of adaptation, in this study, we are most concerned with changes that occur in response to short duration $(0.2-1 \mathrm{~s})$ repetitive stimulation.

The metrics that we collected across the age spectrum could be broadly defined in one of two categories: those that are peripherally biased and those that are predominantly centrally mediated. We predicted that the measures that are predominantly peripherally mediated would be most sensitive to the impact of aging while measures that are predominantly centrally mediated would be less impacted. The results demonstrate that the peripherally mediated measures, such as threshold detection, were - as previously reported by others - significantly impacted with increasing age. This is not surprising, as most of these measures are primarily related to skin physiology, and it is well established that sensory thresholds do increase with age. Centrally mediated measures, such as those that rely mechanistically on cortical information processing properties such as lateral inhibition and/or adaptation, 
however, did not change with age. We viewed this as being consistent with the idea that others (e.g., Dinse, 2006; Greenwood, 2007) have put forth that cortical plasticity is maintained in normal aging and compensates for both anatomical and physiological losses that have been shown to naturally occur with age.

\section{MATERIALS AND METHODS}

In this study, 120 healthy subjects from a wide age spectrum (1870 years) were recruited from the students and employes of the University of North Carolina at Chapel Hill. The subjects were divided into six age groups, 20 subjects in each group. A survey about medication and medical history was filled out by each subject before experimental tests to exclude subjects with a history of neurological impairment. All the subjects were naïve both to the study design and issue under investigation. The study was performed in accordance with Declaration of Helsinki, all subjects gave their written informed consent, and the experimental procedures were reviewed and approved in advance by an institutional review board.

During an experimental session, the subject was seated comfortably in a chair with right arm resting on an arm rest attached to the head unit of a portable four-site vibrotactile stimulator (Figure 1; CM4, Cortical Metrics, LLC). Vibrotactile stimulation was conducted via $5 \mathrm{~mm}$ diameter probes that come in contact with subject's digit 2 (index finger) and digit 3 (middle finger) of the right hand. The independent probe tips are computer controlled and capable of delivery of a wide range of vibrotactile stimulation of varying frequencies (measured in Hertz) and amplitudes (measured in micrometers). Glabrous pads of digit 2 (D2) and digit 3 (D3) were chosen as the test sites for two reasons: (1) to allow the convenience of access and comfort of the subject, and (2) because of the wealth of neurophysiological information that exists for the corresponding somatotopic regions of cortex in primates. The subject's left hand was holding a two-button response device. During each test, the subject was instructed to press the left/right button when the correct stimulus was perceived on the index/middle finger, respectively.

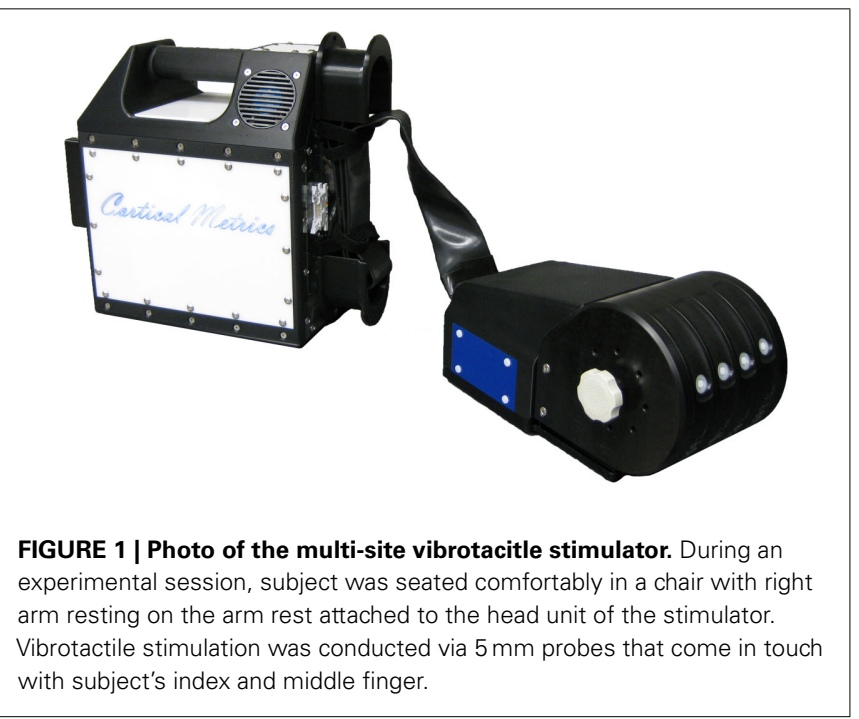

Visual cueing was provided with a computer monitor during the experimental runs. Specifically, an on-screen light panel indicated to the subject when the stimulus was on and when the subject was to respond. An audiometer was used to make sure that no auditory cues were emitted from the stimulator during delivery of the stimuli. Practice trials were performed before each test which allowed the subjects to become familiar with the test, and correct response on five consecutive training trials were required before commencing with each test. The subject was not given performance feedback or knowledge of the results during data acquisition. Stimulus parameters are specified by test algorithms based on specific protocols and subjects' responses during those protocols.

In the current study, a series of metrics were employed to assess each subject's tactile information processing capacity. The total experiment - from start to finish - lasted approximately $30 \mathrm{~min}$ and consisted of the following six metrics: (1) simple reaction time (RT); (2) choice RT; (3) static detection threshold; (4) dynamic detection threshold; (5) amplitude discrimination between two concurrent stimuli; (6) amplitude discrimination after pre-exposure to a conditioning stimulus to one of the stimulus sites (single-site adaptation). Exemplary use, technical description, and neurobiological basis of individual metrics have previously been described in detail (Tannan et al., 2007a,b, 2008; Tommerdahl et al., 2007a; Folger et al., 2008; Francisco et al., 2008; Zhang et al., 2009). An overview of the procedures is provided below.

Simple RT was measured for 14 times during an experimental run for each subject. The left panel of Figure 2A shows the schematic of the protocol. During each trial a single tap (amplitude in $300 \mu \mathrm{m}$ ) was delivered to D2. The subject was instructed to press a response button as soon as the tap was felt. After subject's response, a delay between 2 and $7 \mathrm{~s}$ was placed before the onset of the next trial. For each trial, the RT was recorded as the time interval between stimulation tap and subject's response. In total, 14 simple RTs were obtained for each subject. During the course of data analysis, the two largest and two minimum RT values were excluded in order to eliminate the effects of anticipation and inattention. As a result, a subject's simple RT was calculated as the average of $10 \mathrm{RTs}$ recorded.

Choice RT was measured using a 14-trial two alternative forced choice (2AFC) protocol. The right panel of Figure 2A shows the schematic of the protocol. During each trial a single tap (amplitude in $300 \mu \mathrm{m}$ ) was delivered to either D2 or D3; the stimulus location was randomly selected on a trial-by-trial basis in order to minimize subject's inattention and distraction. The subject was instructed to select the skin site (D2 or D3) that received the tap as fast as possible by pressing the left or right button on the response box. The response accuracy was recorded for each trial. After excluding the two largest and two minimum values, the average response time of trials with correct response was considered as a subject's choice RT. The average performance accuracy of all the subjects is $95 \%$.

\section{STATIC DETECTION THRESHOLD}

Each subject's vibrotactile detection threshold was measured using a 20-trial 2AFC tracking protocol (for recent description with 


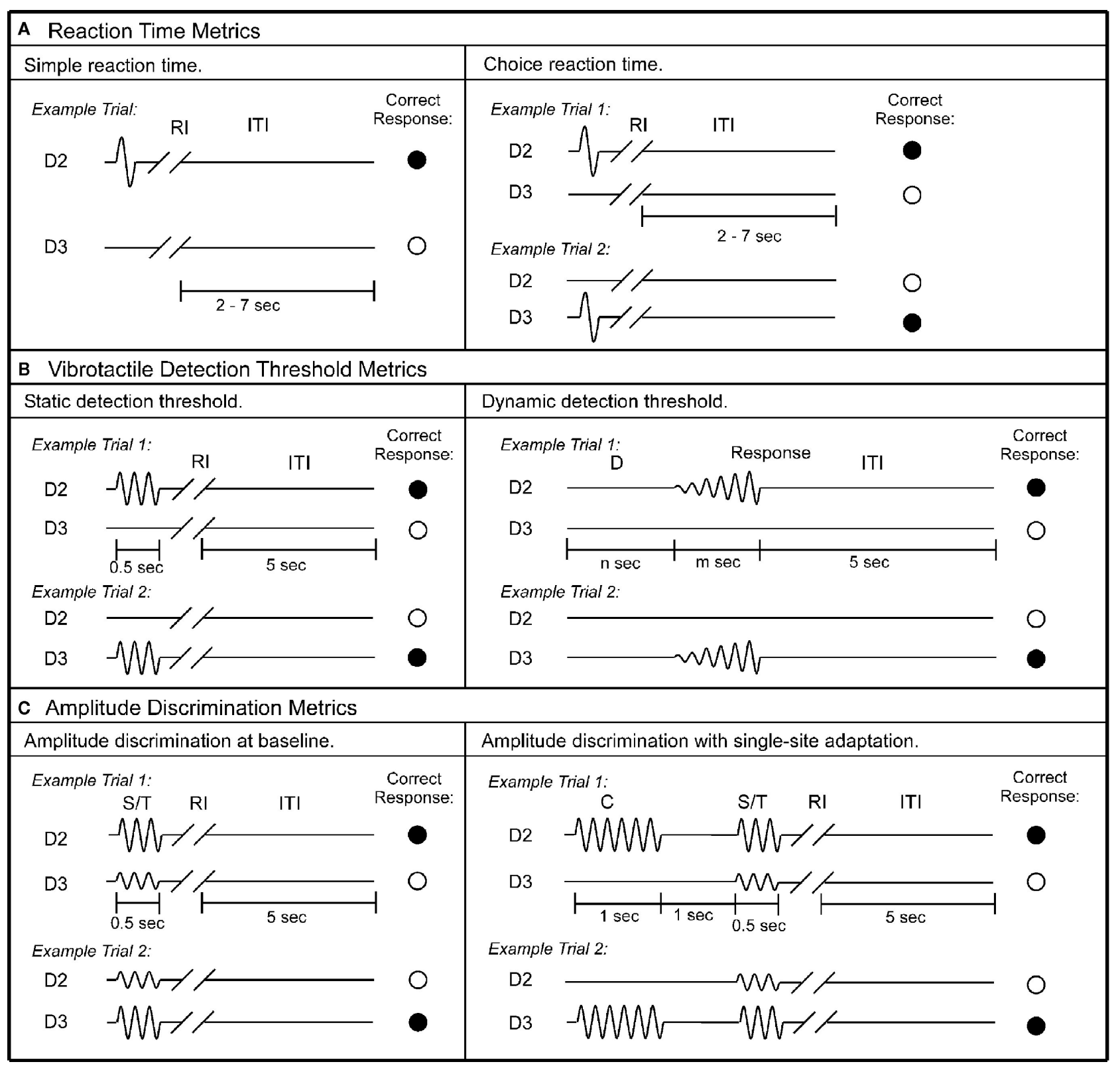

FIGURE 2 | Schematics of the protocols used in this study. (A)

Reaction time metrics. Left panel: during each trial of the simple RT task, a single tap was delivered to D2, followed by response interval (RI).

Subject was instructed to press a response button as soon as the tap was felt. After subject's response, an inter-trial interval (ITI) between 2 and $7 \mathrm{~s}$ was placed before the onset of the next trial. Right panel: during each trial of the choice RT task, a single tap was delivered to either D2 or D3. Subject was instructed to select the skin site that received the tap as fast as possible. (B) Vibrotactile detection threshold metrics. Left panel: during each trial of the static detection threshold task, a $25-\mathrm{Hz}$ vibrotactile test stimulus was delivered to either D2 or D3 for $0.5 \mathrm{~s}$. Subject was instructed to select the skin site that perceived the stimulus. A 5-s ITI intervened between subject's response and onset of the next trial. Right panel: during each trial of the dynamic detection threshold task, a delay period (D) ( $n$ seconds $=0,1.5,2$, or $3 \mathrm{~s}$ ) without any stimulation was applied. After the initial delay, a $25-\mathrm{Hz}$ vibrotactile stimulus was delivered to either D2 or D3. The amplitude of the stimulus was initiated from zero and increased in steps of $2 \mu \mathrm{m} / \mathrm{s}$. The stimulation was terminated with subject response to the perceived stimulus. (C) Amplitude discrimination metrics. Left panel: during each trial of the amplitude discrimination task, two $25 \mathrm{~Hz}$ vibrotactile stimuli - the standard (S) and test (T) - were delivered simultaneously for $0.5 \mathrm{~s}$. Subject was instructed to choose the stimulus that was perceptually larger. Right panel: the amplitude discrimination task was conducted after single-site adaptation. During each trial, a $25-\mathrm{Hz}$ conditioning stimulus (C) was delivered for $1 \mathrm{~s}$ prior to the presentation of the test and standard stimuli. this experiment setup, see previous studies Zhang et al., 2009). The left panel of Figure 2B displays the schematic of the protocol. During each trial a $25-\mathrm{Hz}$ vibrotactile test stimulus (lasts
$500 \mathrm{~ms}$ ) was delivered to either D2 or D3; the stimulus location was randomly selected on a trial-by-trial basis. Following each vibrotactile stimulus, the subject was prompted to select the 
skin site (D2 vs. D3) that perceived the stimulation. After a 5-s delay - based on subject response - the stimulation was repeated until the completion of the 20 trials. The stimulus amplitude was started at $15 \mu \mathrm{m}$ and was modified based on the subject's response in the preceding trial. During the initial 10 trials, a 1up/1-down algorithm was used for the purposes of amplitude modification. For example, the stimulus amplitude was decreased by $1 \mu \mathrm{m}$ if the subject's response in the preceding trial was correct. However, it was increased by $1 \mu \mathrm{m}$ if the response was incorrect. After the initial 10 trials, the amplitude was varied using a 2-up/1-down algorithm (two correct/one incorrect subject response(s) resulted in a decrement/increment, respectively, in the amplitude of the stimulus). The rationale for using 1-up/1down algorithm in the first 10 trials was to expedite determination of subject's vibrotactile discriminative range without affecting the results, and this approach has been previously reported (Tannan et al., 2006, 2007a,b, 2008; Tommerdahl et al., 2007a,b, 2008; Folger et al., 2008; Francisco et al., 2008; Zhang et al., 2008, 2009, 2011).

\section{DYNAMIC DETECTION THRESHOLD}

At the beginning of each trial (as shown in Figure 2B, right panel), a delay period (D) which includes no stimulation was applied. Four conditions of delay ( $n$ seconds) were employed, in separate trials: $0,1.5,2$, and $3 \mathrm{~s}$. After the initial delay, a $25-\mathrm{Hz}$ vibrotactile stimulus was delivered to either D2 or D3 (the stimulus location was randomly selected on a trial-by-trial basis). The amplitude of the stimulus was initiated from zero and increased in steps of $2 \mu \mathrm{m} / \mathrm{s}$. The subject was instructed to indicate the skin site that received the stimulus as soon as the vibration was detected. The stimulus amplitude at the time of subject's response was recorded, and only the value with accurate response was used to calculate the subject's average dynamic detection threshold.

\section{AMPLITUDE DISCRIMINATION AT BASELINE}

Each subject's amplitude discrimination capacity was assessed using a $2 \mathrm{AFC}$ tracking protocol that has been described and implemented in a number of previous studies (Tannan et al., 2007a,b, 2008; Tommerdahl et al., 2007a; Folger et al., 2008; Francisco et al., 2008; Zhang et al., 2008, 2009, 2011). As shown in Figure 2C left panel, during the 20-trial experimental run, a vibrotactile test stimulus (T; $25 \mathrm{~Hz}$, amplitude between 105 and $200 \mu \mathrm{m}$ ) was delivered to one digit pad at the same time that a standard stimulus (S; $25 \mathrm{~Hz}$, amplitude fixed at $100 \mu \mathrm{m}$ ) was applied to the other digit pad. The loci of the test and standard stimuli were randomly selected on a trial-by-trial basis. At the beginning of the experimental run, the test amplitude was $200 \mu \mathrm{m}$ and the standard amplitude was $100 \mu \mathrm{m}$. The difference between the amplitudes of the test and standard stimuli was adjusted on the basis of the subject's response in the preceding trial, such that the difference was decreased/increased after a correct/incorrect response, respectively. The step size was held constant at $10 \mu \mathrm{m}$ throughout the experimental run. The same tracking algorithm as that described for the tactile detection threshold protocol was employed to track the subject's ability to determine the most intense stimulus between the test and standard stimuli [i.e., the subject's difference limen (DL) was determined].

\section{ADAPTATION METRIC}

Amplitude discrimination with single-site adaptation. In order to measure the effects that conditioning stimuli have on subsequent test stimuli, the previously described amplitude discrimination protocol was modified such that delivery of the test and standard stimuli was preceded by a single conditioning stimulus to one of the two stimulus sites (as shown in Figure 2C, right panel). Specifically, a $25 \mathrm{~Hz} 200 \mu \mathrm{m}$ conditioning stimulus (C) was delivered $1 \mathrm{~s}$ prior to the presentation of the test and standard stimuli $(\mathrm{S} / \mathrm{T})$. The duration of the conditioning stimulus was $1 \mathrm{~s}$, which was followed by a 1-s delay before onset of the simultaneous delivery of the test and standard stimuli. The result of such a protocol modification is that the amplitude discrimination DL is typically significantly elevated after pre-exposure to a single-site conditioning stimulation (Tannan et al., 2007b, 2008; Folger et al., 2008; Zhang et al., 2009, 2011). When the conditioning stimulus is delivered to the same site as the test stimulus, the gain effect of adaptation (reducing the perceived intensity) can be quantified by comparison of the DL obtained in the adapted vs. non-adapted conditions (amplitude discrimination at baseline). The tracking algorithm used in the previously described protocol was employed.

\section{ANALYSIS}

One way analysis of variance (ANOVA) and two-sample $t$-test were used to evaluate the difference of the subject's performance across different groups. Data are presented as means and SE. A probability of less than 0.05 was considered statistically significant.

\section{RESULTS}

In the current study, a series of sensory perceptual measures was performed on healthy control subjects of different ages (ranging from 18 to 70 years) that assessed: (1) RT; (2) vibrotactile detection threshold; (3) amplitude discrimination capacity; and (4) the impact of adaptation on amplitude discrimination capacity. The results indicate that although RT and sensory thresholds increased as a function of age, the subject's discriminative capacity and the effects of adaptation on performance remained constant across all the age groups tested.

Reaction time increases with age. Figure $\mathbf{3 A}$ summarizes the group-averaged RT of six age groups. Both choice and simple RTs progressively increase with advancing age. One way ANOVA was performed to compare the mean RT across six age groups, and there is evidence that there are significant differences in the means across groups ( $p<0.001$ for both simple and choice RT). Two-sample $t$-test was employed to compare the mean RT of the subjects under 25 years vs. the mean RT of the subject older than 60 years. There is a significant difference in the mean simple RTs (182 vs. $302 \mathrm{~ms}$ ) and mean choice RTs ( 362 vs. $498 \mathrm{~ms}$ ) with $p<0.001$. The data suggests an age-related decrement in response speed. Note that for all the age groups, choice RT is always higher than simple RT. The difference between choice RT and simple RT might reflect the duration that it takes for a subject to identify a stimulus location. In Figure $\mathbf{3 B}$, the group-averaged RT values are plotted against age. Strong linear relationship (positive correlation) between RTs and age were observed, with $R^{2}=0.99$ for simple RT and $R^{2}=0.95$ for choice RT. 


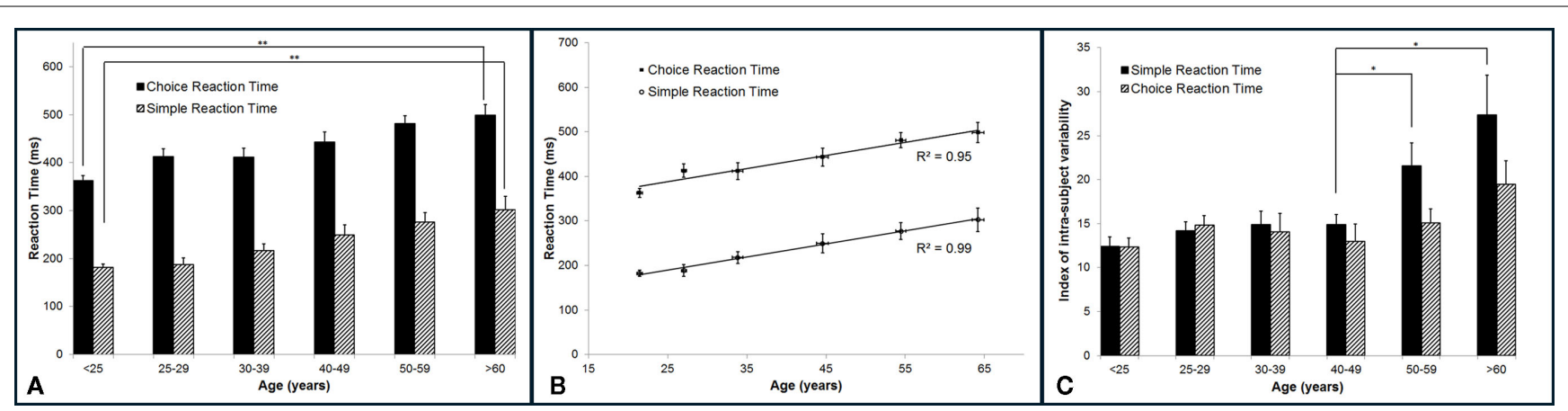

FIGURE 3 | Summary of the group-averaged RTs for six age groups.

(A) Both simple and choice RT progressively increase with advancing age. Comparing the performance between the subjects under 25 years to the subjects older than 60 years, there is a significant difference in the mean simple RT and choice RT with $p<0.001$. (B) A strong linear relationship (positive correlation) between RTs and age was observed, with $R^{2}=0.99$ for simple RT and $R^{2}=0.95$ for choice RT. (C) Summary of the group-averaged index of intra-individual variability for the six age groups Looking at means of intra-individual variability for simple RT, there is no significant difference in the mean across groups that are younger than 50 years $(p=0.4)$. However, significant difference was found between mean of $40-49$ years group and means of $50+$ groups $(p<0.05)$. No significant difference was found for choice RT performance across groups $(p=0.11)$.
In the current study, subjects performed each RT test for 14 times. In order to calculate the index of intra-individual variability, the SD of repeated RT measures was normalized to the mean RT for each subject individually. The group-averaged index of intra-individual variability (\%) on RT performance was calculated and plotted in Figure 3C. One way ANOVA was performed. It was found that there is evidence of significant differences in the means of intra-individual variability for simple RT performance $(p<0.001)$ across six age groups, while no significant differences are found for choice RT performance $(p=0.11)$ across groups. Looking at the intra-individual variability for simple RT by itself, there is no significant differences in the means across age groups younger than 50 years $(p=0.4)$. However, two-sample $t$-test shows significant difference between mean of 40-49 years age group and mean of $50-59$ years age group $(p<0.05)$. The data demonstrates that the group-averaged intra-individual variability remains relatively constant for the subjects younger than 50 years old, while the older subjects ( $>50$ years) have significant higher intra-individual variability.

Vibrotactile detection threshold increases with age. The groupaveraged detection thresholds were obtained with two different methods: a static testing paradigm and a dynamic testing paradigm. As shown in Figure 4A, the group-averaged static threshold gradually increases with advancing age. Specifically, the averaged static threshold for the subjects who are older than 60 years is $13.95 \mu \mathrm{m}$ which is about $8 \mu \mathrm{m}$ larger than that of the subjects under 25 years old $(5.42 \mu \mathrm{m})$. Since several studies have reported that psychophysical measurement methods had a significant influence on vibrotactile threshold (Maeda and Griffin, 1994; Morioka and Griffin, 2002), the threshold was also measured by a dynamic tracking protocol, in which a continuously increasing stimulus was delivered. Following the same trend as observed with static testing paradigm, the group-averaged dynamic threshold progressively rises with aging. In general, the data suggest an elevated tactile sensitivity for older subjects.

It is noteworthy that all subjects demonstrated a dynamic threshold that was higher than their static threshold. This noticeable difference in the threshold between the two tasks is consistent with previous reports (Morioka and Griffin, 2002; Zhang et al., 2009, 2011). One of the explanations could be linked to the fact that dynamic threshold is RT dependent, while static threshold is independent of RT. If this is simply the case, the difference between dynamic and static threshold should be equal to the product of choice RT and the speed of amplitude increment $(2 \mu \mathrm{m} / \mathrm{s})$ during dynamic threshold measurement. Based on this assumption, we calculated the predicted dynamic thresholds using following equation:

\section{Predicted dynamic threshold $=$ \\ Observed static threshold + Choice RT $* 2 \mu \mathrm{m} / \mathrm{s}$}

Figure 4B compares the predicted and observed dynamic thresholds, and the predicted values are always significantly smaller than the observed thresholds, strongly suggesting that the difference between the two measures is not simply due to RT. Figure $4 \mathrm{C}$ is a direct comparison between the two threshold metrics for each age group (actually a ratio of dynamic/static), and it emphasizes not only that the dynamic threshold is always greater than the static threshold, but that this ratio decreases with age. There is a significant difference between the youngest age group and the oldest age group $(p<0.05)$.

Amplitude discrimination capacity and the effects of adaptation were not altered with increases in age. Figure 5 summarizes the group-averaged amplitude discrimination performance obtained during amplitude discrimination task with or without pre-exposure to a conditioning stimulus (adaptation). The data demonstrate that, in the absence of single-site adaptation, subjects were able to discriminate between a $100 \mu \mathrm{m}$ and nearly $125 \mu \mathrm{m}$ stimulus equally well for all the age groups. On the other hand, the delivery of a conditioning stimulus to one of the two stimulus sites prior to the amplitude discrimination task significantly impacted the subject's amplitude discrimination capacity, and the effects of adaptation maintained well across all the age groups. This observed impairment of amplitude discrimination capability 


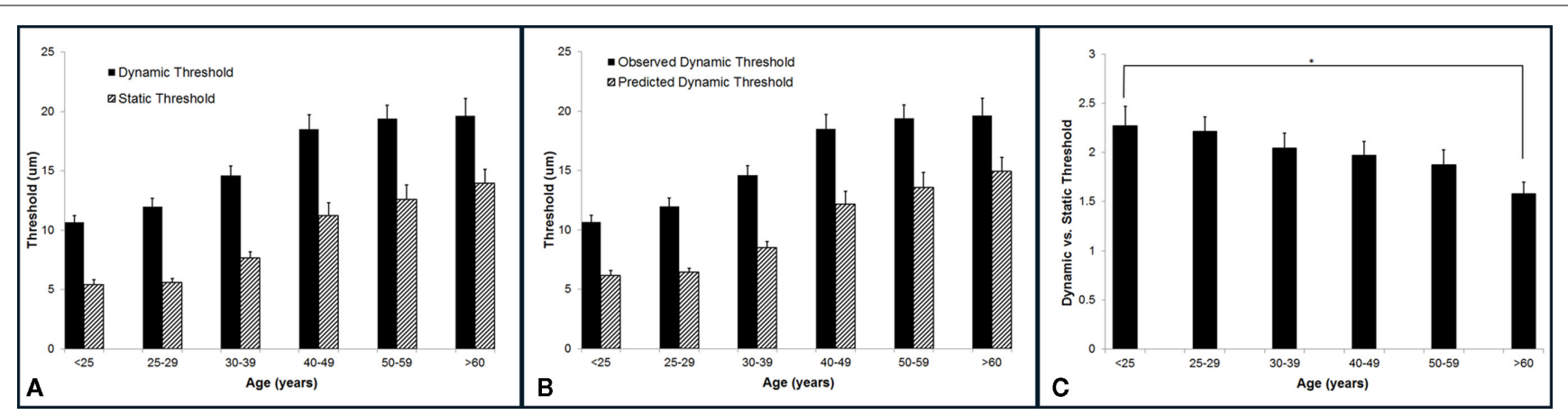

FIGURE 4 | Summary of group-averaged vibrotactile detection thresholds obtained with two different methods across six age groups. (A) Both static and dynamic detection threshold progressively rises with aging. All subjects demonstrated a dynamic threshold that was higher than their static threshold. (B) Comparison of the predicted and observed dynamic thresholds. The predicted values are always significantly smaller than the observed thresholds. (C) Summary of ratio of dynamic vs. static detection threshold across six age groups. Not only the dynamic threshold is always greater than the static threshold, but the dynamic vs. static ratio decreases with age. There is a significant difference between the youngest age group and the oldest age group $(p<0.5)$.

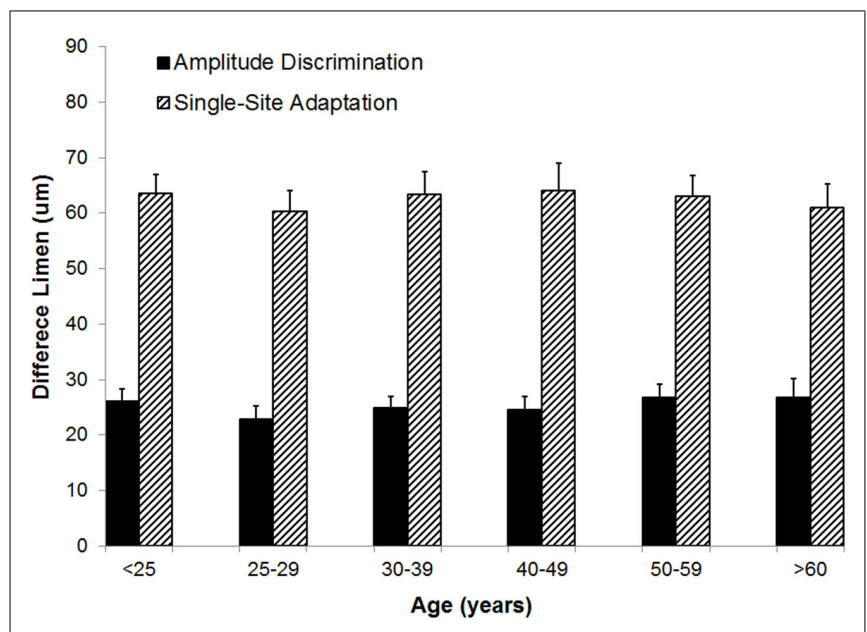

FIGURE 5 | Comparison of difference limen obtained with amplitude discrimination tasks with or without single-site adaptation. There is no significant difference in means across six age groups for both the metric of amplitude discriminative capacity $(p=0.85)$ as well as the adaptation metric $(p=0.98)$.

due to adaptation is consistent with the results of previous studies (Tannan et al., 2007b, 2008; Folger et al., 2008; Zhang et al., 2009). One interpretation of this impairment is that a 1-s conditioning stimulus reduces the perceived intensity of the subsequent test stimulus to the extent that a stimulus with amplitude of approximately $170 \mu \mathrm{m}$ (compared to $125 \mu \mathrm{m}$ without adaptation) was perceived as nearly the same in intensity as the $100 \mu \mathrm{m}$ stimulus. One way ANOVA proves that there is no difference in means across six age groups for the amplitude discrimination task with adaptation $(p=0.98)$ or without adaptation $(p=0.85)$. To summarize the finding across the age spectrum, there is no significant difference in amplitude discrimination performance between subjects of different age groups indiscriminative capacity with or without the presence of single-site conditioning stimuli. In other words, both the metric of amplitude discriminative capacity as well as the adaptation metric (the degree to which amplitude discriminative capacity changed with the conditioning stimulus) were maintained with increases in age.

\section{DISCUSSION}

The present study evaluated the tactile information processing capacity of healthy human subjects across a wide age range (1870 years). Six tests were performed to assess: (1) simple and choice RT; (2) vibrotactile detection thresholds; (3) amplitude discrimination capacity; (4) the effects of adaptation on amplitude discrimination capacity. While the results of peripherally mediated measures demonstrated significant increases in RT and detection threshold with age, the subjects' performance on the centrally mediated measures did not change. Specifically, the amplitude discrimination capacity and the impact of adaptation on performance were maintained with age. If adaptation is a metric that parallels cortical plasticity, the results of the current study suggest that the central nervous system (CNS) in the aging population is still capable of plastic changes, and this cortical plasticity could be the mechanism that compensates for the degradations that are known to naturally occur with age.

Among many cognitive skills, speed of information processing is considered to be especially prone to aging effects. Prior studies have shown a significant increase in RT between 20 and 60 year olds (Fozard et al., 1994; Ratcliff et al., 2001), and this compares favorably with the results obtained in this study. In the current study, the subject's tactile information processing speed was assessed with two well established tasks: simple RT and choice RT tasks. We found that group-averaged RT was positively correlated with the average age for each group, with a correlation coefficient of 0.99 for simple RT and 0.95 for choice RT. Several studies have speculated the reasons for slowing RT with age, and factors other than simple speed of nerve transmission are most often cited. For example, human white matter integrity has been found to significantly correlate with information processing speed (Deary et al., 2006; Madden et al., 2009; Vernooij et al., 2009; Penke et al., 2010). Vernooij et al. (2009) conducted diffusion tensor imaging (DTI) scans and cognitive tasks in a sample of 860 older adults 61-92 years of 
age. It has been found that performance on tests that rely on processing speed degrades significantly with declining white matter integrity of the whole brain. Since many of these studies were performed on older healthy subjects without signs of mild cognitive impairment or dementia, the increase of RT might simply represent the effects of normal aging on basic cognitive function. In the context of the current study, we speculate that the increased mean RT could be the result of both decreased nerve transmission speed with age as well as the age-related decline in white matter integrity.

Increases in intra-individual variability on RT performance have been observed for older subjects compared with younger subjects. For example, it has been shown that inconsistency across trials on RT performance increases with age (Hultsch et al., 2000, 2002; Gorus et al., 2008; Bunce et al., 2010). In this report, we found that while the group-averaged intra-individual variability remains relatively constant for the subjects younger than 50 years old, the older subjects ( $>50$ years) have significant higher intra-individual variability. In other words, older subjects showed greater inconsistency than younger subjects in response speed. Several studies have demonstrated that performance variability has the potential to be a good indicator of neurological disturbance and may be a good marker of preclinical status of dementia. For example, Bunce et al. (2010) found greater frontal white matter lesions were associated with higher intra-individual variability in choice RT in middleaged healthy adults. Hultsch et al. (2000) also demonstrated that performance variability was greater in patients with mild dementia than in healthy elderly subjects. As a result, measures of intraindividual variability may be a plausible behavioral indicator of aging-induced central neurological disturbances and may be able to serve as a valuable early marker of neurodegenerative disease.

Tactile detection threshold (a measure which determines the minimum stimulus intensity that can be perceived), has been documented to increase (due to decreased sensitivity) with age (Verrillo, 1977, 1979, 1980; Thornbury and Mistretta, 1981; Kenshalo, 1986; Gescheider et al., 1994; Lin et al., 2005). In the current study, the data is consistent with prior observations and shows degraded vibrotactile sensitivity (at $25 \mathrm{~Hz}$ ) with increasing age. In order to determine if mechanisms involved in processing sub-threshold vs. threshold stimuli could be differentiated, tactile detection thresholds were collected using two different protocols. "Static" threshold is the minimum constant-amplitude stimulus detected, and "dynamic" threshold refers to the detection threshold measured with a stimulus that is increased from zero intensity to a detectable level (Zhang et al., 2009, 2011). It is noteworthy that all subjects demonstrated a dynamic threshold that was higher than their static threshold. This noticeable difference in the threshold between the two tasks is consistent with previous reports (Morioka and Griffin, 2002; Zhang et al., 2009, 2011). Since an argument could be made that the primary difference between the two measures is one of RT - dynamic threshold is RT dependent, while static threshold is independent of RT - we directly compared the actual results vs. results predicted based on this RT difference. As demonstrated in Figure $4 \mathrm{~B}$ of Results, the difference between the observations obtained by the two methods could not be explained by RT alone. An alternative possibility - and one that the authors have recently proposed (Tommerdahl et al., 2010; Favorov and Kursun, 2011; Zhang et al., 2011) - is that the difference between the two threshold metrics is impacted significantly by feed-forward inhibition that is generated by the initial sub-threshold stimulus that occurs when the dynamic threshold test is ramped from a null to a detectable level. Thus, the sub-threshold stimulus delivered by the dynamic threshold test actually leads to the initial inhibition, or adaptation, that ultimately requires a larger stimulus to reach detectable levels.

One of the interesting findings of the current study is that although the subjects' vibrotactile detection threshold went up with age, their amplitude discrimination capacity was maintained. Specifically, subjects in all age groups demonstrated a similar ability to differentiate two supra-threshold stimuli that are delivered simultaneously to the skin. It should be noted that this amplitude discrimination task was conducted at supra-threshold levels (approximately $10 \times$ normative thresholds), and all subjects had approximately the same amplitude discriminative capacity at the amplitudes used. Thus, while the decline of tactile sensitivity is considered to be influenced predominantly by peripheral factors, we speculate that the ability to discriminate between two supra-threshold stimuli is more influenced by centrally mediated factors and would be only moderately influenced by changes in the periphery. This hypothesis was derived, in part, from studies which demonstrated that localized increases in the magnitude of the SI cortical response (Simons et al., 2005, 2007; Friedman et al., 2008) paralleled the changes in the ability of human subjects to distinguish between different intensities of skin stimulation (i.e., amplitude discrimination; Francisco et al., 2008).

To investigate potential changes in cortical plasticity with normal aging, the effect of single-site adaptation on amplitude discrimination capacity was measured. Previous studies using this adaptation metric demonstrated that a conditioning stimulus delivered to one of the two sites before the amplitude discrimination task significantly altered a subject's ability to determine the actual difference between the two stimuli (Tannan et al., 2007b, 2008) by introducing a confound. In other words, the conditioning stimulus makes the subsequent stimulus, at the conditioned site, feel weaker and consequently, amplitude discriminative capacity is reduced. Neurophysiological studies have demonstrated that the effects of reduced intensity due to adapting stimulation are possibly attributable to a reduction in the responsivity of central neurons after prolonged or repetitive stimulation (Lee and Whitsel, 1992; Lee et al., 1992). When the single-site adaptation measure is examined across a number of subject populations with compromised CNS - as may be the case with a neurodevelopmental disorder: autism (Tannan et al., 2008), acute pharmacological block (Folger et al., 2008), or a chronic pain condition (Zhang et al., 2011) - the adaptation metric is significantly diminished from that of the control population. These findings suggest that the method could be viewed as a potential indicator or marker of systemic cortical alterations, as adaptation, at this short duration time scale, is impacted by a number of factors (for discussion, see Tannan et al., 2007b, 2008; Tommerdahl et al., 2007a, 2010; Folger et al., 2008; Francisco et al., 2008; Zhang et al., 2009, 2011).

Evidence from a wide range of studies has demonstrated that while there are aspects of anatomical and functional degradation with age, the CNS is still capable of plastic changes. For instance, in a series of studies, Dinse and colleagues reported 
that experimental or environmental stimulations could induce use-dependent plasticity in older animal as well as human subjects at both cortical and behavioral level (Hilbig et al., 2002; Li and Dinse, 2002; Dinse, 2005, 2006; Dinse et al., 2006; Kalisch et al., 2008, 2009; Kattenstroth et al., 2010). Specifically, it has been found that aged rats exposed to an enriched environment showed complete recovery from the age-related enlargement of RFs of the hindpaw in somatosensory cortex typically found in animals housed in standard conditions (Hilbig et al., 2002). At the behavioral level, repetitive sensory stimulation procedures resulted in improvement of tactile acuity in elderly individuals, a phenomenon based on synaptic plasticity (Dinse, 2005; Dinse et al., 2006). In this study, we found that the effects of adaptation

\section{REFERENCES}

Bartzokis, G., Cummings, J. L., Sultzer, D., Henderson, V. W., Nuechterlein, K. H., and Mintz, J. (2003). White matter structural integrity in healthy aging adults and patients with Alzheimer disease: a magnetic resonance imaging study. Arch. Neurol. 60, 393-398.

Bunce, D., Anstey, K. J., Cherbuin, N., Burns, R., Christensen, H., Wen, W., and Sachdev, P. S. (2010). Cognitive deficits are associated with frontal and temporal lobe white matter lesions in middle-aged adults living in the community. PLoS ONE 5, el3567. doi:10.1371/journal.pone. 0013567

Deary, I. J., Bastin, M. E., Pattie, A., Clayden, J. D., Whalley, L. J., Starr, J. M., and Wardlaw, J. M. (2006). White matter integrity and cognition in childhood and old age. Neurology $66,505-512$.

Dinse, H. R. (2005). Treating the aging brain: cortical reorganization and behavior. Acta Neurochir. Suppl. 93, 79-84.

Dinse, H. R. (2006). Cortical reorganization in the aging brain. Prog. Brain Res. 157, 57-80.

Dinse, H. R., Kleibel, N., Kalisch, T., Ragert, P., Wilimzig, C., and Tegenthoff, M. (2006). Tactile coactivation resets age-related decline of human tactile discrimination. Ann. Neurol. 60, 88-94.

Driscoll, I., Davatzikos, C., An, Y., Wu, X., Shen, D., Kraut, M., and Resnick, S. M. (2009). Longitudinal pattern of regional brain volume change differentiates normal aging from MCI. Neurology 72, 1906-1913.

Favorov, O. V., and Kursun, O. (2011). Neocortical layer 4 as a pluripotent function linearizer. J. Neurophysiol. 105, 1342-1360.

Fjell, A. M., Walhovd, K. B., FennemaNotestine, C., Mcevoy, L. K., Hagler, D. J., Holland, D., Brewer, J. B., and
Dale, A. M. (2009). One-year brain atrophy evident in healthy aging. $J$. Neurosci. 29, 15223-15231.

Folger, S. E., Tannan, V., Zhang, Z., Holden, J. K., and Tommerdahl, M. (2008). Effects of the $N$ methyl-D-Aspartate receptor antagonist dextromethorphan on vibrotactile adaptation. BMC Neurosci. 9, 87. doi:10.1186/1471-2202-9-87

Fozard, J. L., Vercryssen, M., Reynolds, S. L., Hancock, P. A., and Quilter, R. E. (1994). Age differences and changes in reaction time: the Baltimore Longitudinal Study of Aging. J. Gerontol. 49, P179-P189.

Francisco, E., Tannan, V., Zhang, Z., Holden, J., and Tommerdahl, M. (2008). Vibrotactile amplitude discrimination capacity parallels magnitude changes in somatosensory cortex and follows Weber's Law. Exp. Brain Res. 191, 49-56.

Friedman, R. M., Chen, L. M., and Roe, A. W. (2008). Responses of areas $3 \mathrm{~b}$ and 1 in anesthetized squirrel monkeys to single- and dual-site stimulation of the digits. J. Neurophysiol. 100, 3185-3196.

Gescheider, G. A., Bolanowski, S. J., Hall, K. L., Hoffman, K. E., and Verrillo, R. T. (1994). The effects of aging on information-processing channels in the sense of touch: I. Absolute sensitivity. Somatosens. Mot. Res. 11, 345-357.

Gorus, E., De Raedt, R., Lambert, M., Lemper, J. C., and Mets, T. (2008). Reaction times and performance variability in normal aging, mild cognitive impairment, and Alzheimer's disease. J. Geriatr. Psychiatry Neurol. 21, 204-218.

Greenwood, P. M. (2007). Functional plasticity in cognitive aging: review and hypothesis. Neuropsychology 21 , 657-673.

Greenwood, P. M., and Parasuraman, R. (2010). Neuronal and cognitive plasticity: a neurocognitive

remain relatively constant across healthy populations regardless of age. Since adaptation is an important feature of cortical information processing that apparently remains intact with normal aging, it could be an important feature to assess in the aging population. Deviations from normative values could be an early indicator of neurodegenerative disease; studies directly addressing this issue are currently ongoing and will be reported in the near future.

\section{ACKNOWLEDGMENTS}

This work was supported, in part by the Department of Defense, Congressionally Directed Medical Research Program (CDMRP) W81XWH-07-1-0287, and NIH 1-R21-NS072811-01A1.

framework for ameliorating cognitive aging. Front. Aging Neurosci. 2:150. doi:10.3389/fnagi.2010.00150

Gunning-Dixon, F. M., Brickman, A. M., Cheng, J. C., and Alexopoulos, G. S. (2009). Aging of cerebral white matter: a review of MRI findings. Int. J. Geriatr. Psychiatry 24, 109-117.

Gunning-Dixon, F. M., and Raz, N. (2000). The cognitive correlates of white matter abnormalities in normal aging: a quantitative review. Neuropsychology 14, 224-232.

Hilbig, H., Bidmon, H. J., Steingruber, S., Reinke, H., and Dinse, $\mathrm{H}$. R. (2002). Enriched environmental conditions reverse age-dependent gliosis and losses of neurofilaments and extracellular matrix components but do not alter lipofuscin accumulation in the hindlimb area of the aging rat brain. J. Chem. Neuroanat. 23, 199-209.

Hultsch, D. F., Macdonald, S. W., and Dixon, R. A. (2002). Variability in reaction time performance of younger and older adults. J. Gerontol. B Psychol. Sci. Soc. Sci. 57, P101P115.

Hultsch, D. F., Macdonald, S. W. Hunter, M. A., Levy-Bencheton, J., and Strauss, E. (2000). Intraindividual variability in cognitive performance in older adults: comparison of adults with mild dementia, adults with arthritis, and healthy adults. Neuropsychology 14, 588-598.

Kalisch, T., Ragert, P., Schwenkreis, P., Dinse, H. R., and Tegenthoff, M. (2009). Impaired tactile acuity in old age is accompanied by enlarged hand representations in somatosensory cortex. Cereb. Cortex 19, 1530-1538.

Kalisch, T., Tegenthoff, M., and Dinse, H. R. (2008). Improvement of sensorimotor functions in old age by passive sensory stimulation. Clin. Interv. Aging 3, 673-690.

Kattenstroth, J. C., Kolankowska, I., Kalisch, T., and Dinse, H. R. (2010).
Superior sensory, motor, and cognitive performance in elderly individuals with multi-year dancing activities. Front. Aging Neurosci. 2:31. doi:10.3389/fnagi.2010.00031

Kenshalo, D. R. Sr. (1986). Somesthetic sensitivity in young and elderly humans. J. Gerontol. 41, 732-742.

Kohn, A. (2007). Visual adaptation: physiology, mechanisms, and functional benefits. J. Neurophysiol. 97, 3155-3164.

Lee, C. J., and Whitsel, B. L. (1992). Mechanisms underlying somatosensory cortical dynamics: I. In vivo studies. Cereb. Cortex 2, 81-106.

Lee, C. J., Whitsel, B. L., and Tommerdahl, M. (1992). Mechanisms underlying somatosensory cortical dynamics: II. In vitro studies. Cereb. Cortex 2, 107-133.

Li, S. C., and Dinse, H. R. (2002). Aging of the brain, sensorimotor, and cognitive processes. Neurosci. Biobehav. Rev. 26, 729-732.

Lin, Y. H., Hsieh, S. C., Chao, C. C., Chang, Y. C., and Hsieh, S. T. (2005). Influence of aging on thermal and vibratory thresholds of quantitative sensory testing. J. Peripher. Nerv. Syst. 10, 269-281.

Madden, D. J., Bennett, I. J., and Song, A. W. (2009). Cerebral white matter integrity and cognitive aging: contributions from diffusion tensor imaging. Neuropsychol. Rev. 19, 415-435.

Maeda, S., and Griffin, M. J. (1994). A comparison of vibrotactile thresholds on the finger obtained with different equipment. Ergonomics 37, 1391-1406.

Morioka, M., and Griffin, M. J. (2002). Dependence of vibrotactile thresholds on the psychophysical measurement method. Int. Arch. Occup. Environ. Health 75, 78-84.

Morse, C. K. (1993). Does variability increase with age? An archival study of cognitive measures. Psychol. Aging 8, 156-164. 
Penke, L., Munoz Maniega, S., Murray, C., Gow, A. J., Hernandez, M. C., Clayden, J. D., Starr, J. M., Wardlaw, J. M., Bastin, M. E., and Deary, I. J. (2010). A general factor of brain white matter integrity predicts information processing speed in healthy older people. J. Neurosci. 30, 7569-7574.

Ratcliff, R., Thapar, A., and Mckoon, G. (2001). The effects of aging on reaction time in a signal detection task. Psychol. Aging 16, 323-341.

Raz, N., Lindenberger, U., Rodrigue, K. M., Kennedy, K. M., Head, D., Williamson, A., Dahle, C., Gerstorf, D., and Acker, J. D. (2005). Regional brain changes in aging healthy adults: general trends, individual differences and modifiers. Cereb. Cortex 15, 1676-1689.

Resnick, S. M., Pham, D. L., Kraut, M. A., Zonderman, A. B., and Davatzikos, C. (2003). Longitudinal magnetic resonance imaging studies of older adults: a shrinking brain. J. Neurosci. 23, 3295-3301.

Simons, S. B., Chiu, J., Favorov, O. V., Whitsel, B. L., and Tommerdahl, M. (2007). Durationdependent response of SI to vibrotactile stimulation in squirrel monkey. J. Neurophysiol. 97, 2121-2129.

Simons, S. B., Tannan, V., Chiu, J., Favorov, O. V., Whitsel, B. L., and Tommerdahl, M. (2005). Amplitude-dependency of response of SI cortex to flutter stimulation. BMC Neurosci. 6, 43. doi:10.1186/1471-2202-6-43

Tannan, V., Dennis, R., and Tommerdahl, M. (2005a). A novel device for delivering two-site vibrotactile stimuli to the skin. J. Neurosci. Methods 147, 75-81.
Tannan, V., Dennis, R. G., and Tommerdahl, M. (2005b). Stimulusdependent effects on tactile spatial acuity. Behav. Brain Funct. 1, 18.

Tannan, V., Dennis, R. G., Zhang, Z., and Tommerdahl, M. (2007a). A portable tactile sensory diagnostic device. J. Neurosci. Methods 164, 131-138.

Tannan, V., Simons, S., Dennis, R. G., and Tommerdahl, M. (2007b). Effects of adaptation on the capacity to differentiate simultaneously delivered dual-site vibrotactile stimuli. Brain Res. 1186, 164-170.

Tannan, V., Holden, J. K., Zhang, Z., Baranek, G. T., and Tommerdahl, M. A. (2008). Perceptual metrics of individuals with autism provide evidence for disinhibition. Autism Res. 1, 223-230.

Tannan, V., Whitsel, B. L., and Tommerdahl, M. A. (2006). Vibrotactile adaptation enhances spatial localization. Brain Res. 1102, 109-116.

Thornbury, J. M., and Mistretta, C. M. (1981). Tactile sensitivity as a function of age. J. Gerontol. 36, 34-39.

Tommerdahl, M., Favorov, O. V., and Whitsel, B. L. (2010). Dynamic representations of the somatosensory cortex. Neurosci. Biobehav. Rev. 34 160-170.

Tommerdahl, M., Tannan, V., Cascio, C. J., Baranek, G. T., and Whitsel, B. L. (2007a). Vibrotactile adaptation fails to enhance spatial localization in adults with autism. Brain Res. 1154, 116-123.

Tommerdahl, M., Tannan, V., Zachek, M., Holden, J. K., and Favorov, O. V. (2007b). Effects of stimulus-driven synchronization on sensory perception. Behav. Brain Funct. 3, 61 .

Tommerdahl, M., Tannan, V., Holden, J. K., and Baranek, G. T. (2008).
Absence of stimulus-driven synchronization effects on sensory perception in autism: evidence for local underconnectivity? Behav. Brain Funct. 4, 19.

Van Petten, C., Plante, E., Davidson, P. S., Kuo, T. Y., Bajuscak, L., and Glisky, E. L. (2004). Memory and executive function in older adults: relationships with temporal and prefrontal gray matter volumes and white matter hyperintensities. Neuropsychologia 42, 1313-1335.

Vernooij, M. W., Ikram, M. A., Vrooman, H. A., Wielopolski, P. A., Krestin, G. P., Hofman, A., Niessen, W. J., Van Der Lugt, A., and Breteler, M. M (2009). White matter microstructural integrity and cognitive function in a general elderly population. Arch. Gen. Psychiatry 66, 545-553.

Verrillo, R. T. (1977). Comparison of child and adult vibrotactile thresholds. Bull. Psychon. Soc. 9, 197-200.

Verrillo, R. T. (1979). Change in vibrotactile thresholds as a function of age. Sens. Processes 3, 49-59.

Verrillo, R. T. (1980). Age related changes in the sensitivity to vibration. J. Gerontol. 35, 185-193.

Verrillo, R. T. (1982). Effects of aging on the suprathreshold responses to vibration. Percept. Psychophys. 32, 61-68.

Verrillo, R. T., Bolanowski, S. J., and Gescheider, G. A. (2002). Effect of aging on the subjective magnitude of vibration. Somatosens. Mot. Res. 19, 238-244.

Wilson, R. S., Beckett, L. A., Barnes, L. L. Schneider, J. A., Bach, J., Evans, D. A., and Bennett, D. A. (2002). Individual differences in rates of change in cognitive abilities of older persons. Psychol. Aging 17, 179-193.
Zhang, Z., Francisco, E. M., Holden, J. K., Dennis, R. G., and Tommerdahl, M. (2009). The impact of non-noxious heat on tactile information processing. Brain Res. 1302, 97-105.

Zhang, Z., Tannan, V., Holden, J. K., Dennis, R. G., and Tommerdahl, M. (2008). A quantitative method for determining spatial discriminative capacity. Biomed. Eng. Online 7, 12. doi:10.1186/1475-925X-7-12

Zhang, Z., Zolnoun, D. A., Francisco, E. M., Holden, J. K., Dennis, R. G., and Tommerdahl, M. (2011). Altered central sensitization in subgroups of women with vulvodynia. Clin. J. Pain 27, 755-763.

Conflict of Interest Statement: The authors declare that the research was conducted in the absence of any commercial or financial relationships that could be construed as a potential conflict of interest.

Received: 01 October 2011; accepted: 21 November 2011; published online: 08 December 2011.

Citation: Zhang Z, Francisco EM, Holden JK, Dennis RG and Tommerdahl M (2011) Somatosensory information processing in the aging population. Front. Ag. Neurosci. 3:18. doi 10.3389/fnagi.2011.00018

Copyright (c) 2011 Zhang, Francisco, Holden, Dennis and Tommerdahl. This is an open-access article distributed under the terms of the Creative Commons Attribution Non Commercial License, which permits non-commercial use, distribution, and reproduction in other forums, provided the original authors and source are credited. 